\title{
DNA aneuploidy by flow cytometry is an independent prognostic factor in gastric cancer
}

\author{
Mar Abad ${ }^{\text {a,* }}$, Juana Ciudad ${ }^{\text {d }}$, Manuel R. Rincon ${ }^{\text {c }}$, Isabel Silva ${ }^{\text {b }}$, José I. Paz-Bouza a \\ Antonio Lopez ${ }^{\mathrm{d}}$, Alberto G. Alonso ${ }^{\mathrm{b}}$, Agustin Bullon ${ }^{\mathrm{a}}$ and Alberto Orfao ${ }^{\mathrm{d}}$ \\ ${ }^{a}$ Department of Pathology, University Hospital, Salamanca, Spain \\ ${ }^{\mathrm{b}}$ Department of Surgey, University Hospital, Salamanca, Spain \\ ${ }^{\mathrm{c}}$ Unit of Gastroenterology, University Hospital, Salamanca, Spain \\ ${ }^{\mathrm{d}}$ Servicio de Citometria, Universidad de Salamanca, Salamanca, Spain
}

Received 13 October 1997

Revised 12 March 1998

Accepted 14 April 1998

\begin{abstract}
In the present study the prognostic value of both DNA ploidy and the proliferative activity of tomour cells were studied in a series of 76 consecutive patients suffering from gastric tumours. DNA ploidy and the proliferative index (as measured by the percentage of S-phase cells) were determined by flow cytometry using fresh tumour specimens.

The presence of DNA aneuploid clones by flow cytometry was detected in $62 \%$ of the cases (mean DNA index of $1.63 \pm 0.46$; range 1.08-2.92), the mean proportion of S-phase cells being of $18.4 \pm 11.5 \%$. In comparison with diploid cases, aneuploid tumours showed a higher proliferative activity (cases with more than $15 \%$ S-phase cells: $18.4 \%$ versus $6.1 \%, p=0.0001$ ) as well as a higher incidence of node involvement $(95 \%$ versus $68 \%, p=0.001)$. By contrast, no significant differences were detected with respect to sex, age, histologic grade and type, clinical stage, tumour size and the incidence of extranodal involvement.

Upon grouping the patients according to the proportion of S-phase cells no significant differences were observed for the clinical and biological parameters explored except for an association between a high percentage of S-phase cells and the presence of DNA aneuploidy ( $40 \%$ versus $96 \%, p=0.0001)$.

Regarding survival the presence of DNA aneuploidy was significantly associated with poor outcome as compared to the diploid cases (median of 15 versus 26 months, $p=0.005$ ). By contrast, the proportion of S-phase cells did not predict patients's outcome.

Multivariate analysis of prognostic factors showed that the presence of DNA aneuploidy $(p=0.003)$ together with the histologic type $(p=0.03)$ and the existence of extranodal metastases $(p=0.05)$ were the best combination of prognostic factors for survival prediction.
\end{abstract}

Keywords: DNA aneuploidy, flow cytometry, gastric cancer, prognosis

\section{Introduction}

The investigation of prognostic factors is an essential step in the management of cancer patients. During the last decades several prognostic factors that contribute to improve the prediction of disease outcome have been reported in gastric cancer patients. Among others, these include tumour size, histologic

\footnotetext{
${ }^{*}$ Correspondence to: Dr. Mar Abad, Departamento de Anatomia Patologica, Hospital Clinico Universitario, Paseo de San Vicente s/n, 37007 Salamanca, Spain. Fax: 3423294624.
} 
type, node involvement and extranodal metastases [5,10,22,32]. However, in spite of this an important prognostic heterogeneity still exists even within patients with similar disease characteristics. This points to the need to investigate clinical and biological features that may contribute to a better assessment of the prognosis of the disease.

Measurement of tumour cell DNA content using flow cytometry provides rapid and objective information on both the presence of clonal abnormalities in cell DNA contents (DNA aneuploidy) and the cell cycle distribution of the tumour cell population. Over the last two decades, this type of measurement has been performed in a wide variety of human tumours [30], including gastric cancer. Nevertheless, disturbing levels of variability are found regarding the incidence of both DNA aneuploidy and the proliferative rate of tumour cells and its clinical significance. Thus, the incidence of DNA aneuploidy in gastric cancer ranges between 34\% [2] and 89\% [31] of the cases, depending on the series. Likewise, the mean percentage of S-phase cells in gastric cancer has been reported to be as low as $9 \%$ [3] and as high as $45.1 \%$ as assessed using immunocytochemistry techniques [17]. The reasons for such a variability were evaluated in a DNA Cytometry Consensus Conference held in October 1992 [30]. At this conference, it was concluded that whereas some discrepancies can be related to poorly designed studies lacking sufficient follow-up or number of patients, on many occasions variability could be due to technical artefacts, and new technically sound studies were encouraged [30]. This could also contribute to explain the contradictory results found regarding the clinical value of both the presence of DNA aneuploidy and the proportion of S-phase cells in gastric cancer [6,13,18,19,24,25,37].

In the present study, we prospectively analyzed the prognostic value of both the presence of DNA aneuploidy and the cell cycle distribution of tumour cells as compared to other well-established prognostic factors in a series of 76 consecutive patients diagnosed with gastric cancer using an objective and highly reproducible flow cytometry method.

\section{Material and methods}

\subsection{Patients}

A total of 76 consecutive patients (47 males and 29 females, mean age of $70 \pm 10$ years old) diagnosed with gastric carcinoma was included in the present study. Each case was histologically classified according differentiation criteria (well, moderate and poor). In addition, the patients were also classified on the basis of the existence of neoplastic involvement of the gastric wall (T1: involvement of the lamina propia or submucosa; T2: infiltration of muscularis propria or subserosa; T3: penetration of the visceral peritoneum without invasion of adjacent structures; and T4: invasion of adjacent structures) as well as to the presence of both lymph node involvement (N0: negative lymph nodes; N1: involvement of juxtagastric lymph nodes; and N2: neoplastic infiltration of lymph nodes distant from the primary tumour) and extranodal metastases. The pathologic stage of the cancer was established according to the American Joint Committee on Cancer Criteria [1]. As the moment of closing the study, $48 \%$ of the patients were alive and 52\% had died. The median follow-up for the whole series was 43 months.

\subsection{Flow cytometry DNA analysis}

DNA measurements were performed in all cases at the moment of diagnosis. Tumour samples were obtained by endoscopic biopsies and/or gastric surgery and prepared within $30 \mathrm{~min}$ after being obtained. In both cases, specimens from different tumour regions were pooled, placed on a gauze and cut into small 
pieces of around $1 \times 1 \mathrm{~mm}^{3}$. Tumour pieces were then placed in citrate buffer and smoothly minced with tweezers and pinzars. After allowing the preparations to sediment for one minute, the supernatant was collected, centrifuged and resuspended at a concentration of $5 \times 10^{6}$ cells $/ \mathrm{ml}$ in citrate buffer. Then, $200 \mu \mathrm{l}$ containing approximately $10^{6}$ cells were placed in a separate tube and used to measure cell DNA content according to the technique of Vindelov et al. [26,34]. Briefly, $1.8 \mathrm{ml}$ of a solution containing trypsin $0.03 \mathrm{~g} / \mathrm{l}$ (Sigma), pH 7.6 - was added to the cell suspension. Then, cells were incubated for $10 \mathrm{~min}$ at room temperature. Once this incubation period was finished, $1.5 \mathrm{ml}$ of a solution containing a trypsin inhibitor $-0.1 \mathrm{~g} / \mathrm{l}$ (Sigma) - and RNAse - $0.5 \mathrm{~g} / \mathrm{l}$ (Sigma) - was added and another incubation period of $10 \mathrm{~min}$ was carried out at room temperature. Finally, a third incubation (room temperature) for at least $15 \mathrm{~min}$ was carried out in the dark after adding $1.5 \mathrm{ml}$ of a solution containing propidium iodide $-0.21 \mathrm{~g} / \mathrm{l}$ (Sigma). Measurements were made within three hours after this last incubation had finished. Information on at least $10^{4}$ nuclei was acquired using a FACScan flow cytometer (Becton/Dickinson, San José, CA, USA) equipped with a $15 \mathrm{~mW}$ argon ion laser tuned at $488 \mathrm{~nm}$ and a doublet discrimination module. The electronics of the instrument were adjusted using chicken erythrocyte nuclei so that the modal channel for the diploid G0/G1 nuclei would be 200 (propidium iodide fluorescence intensity was scaled from channel 0 to 1023). The existence of DNA aneuploidy was defined as the existence of two G0/G1 peaks in the DNA histogram according to well-established criteria [8]. DNA indices were calculated by dividing the modal channel obtained for the G0/G1 tumour cells by the modal channel for normal diploid G0/G1 cells present in the sample. Whenever it was not clear which G0/G1 peak corresponded to the tumour population, a second tube in which a mixture of tumour cells and diploid peripheral blood leucocytes from a sex-matched healthy individual was stained with propidium iodide using the technique described above and measured under the same conditions.

Analysis of the distribution of G0/G1, S and G2/M cell cycle phases was performed on gated cells for the exclusion of doublets using the RFIT mathematical calculation model by means of the CellFIT software (Becton/Dickinson). In all samples, where there was an overlap between diploid and aneuploid cell populations, the POLY mathematical calculation model was used to calculate their cell cycle distribution separately. The overall mean coefficient of variation of the G0/G1-phase peak was $3.18 \pm 1.01 \%$ and $2.57 \pm 1.21 \%$ for the diploid and aneuploid populations, respectively. In all cases, the percentage of events present in the DNA histogram that corresponded to background aggregates and death cells was less than $8 \%$, and they could be clearly gated out on a FSC/FLZA dot plot.

\subsection{Statistical methods}

Mean values and their standard deviations were calculated for all variables in each group of patients (BMDP 2D software program). Cut-off points for S-phase were established at those which showed the highest discriminative value for overall survival after testing the median, mean and tertile values. In order to establish the statistical significance of the differences observed between groups, the Mann-Whitney $U$-test was used (BMDP 3S software program).

Survival curves were plotted according to the method of Kaplan and Meier, and the Mantel-Cox, Breslow and Tarone tests were used to establish the statistical significance of the differences observed between curves (BMDP 1L software program). Multivariate analysis was performed to examine the simultaneous effect of the different variables on survival by the stepwise proportional hazards regression model for censored survival data (BMDP $2 \mathrm{~L}$ software program). Variables included in the regression analysis were those for which there was a significant association with survival in the univariate analysis of prognostic factors $(p<0.1)$. 


\section{Results}

From the 76 patients included in the present study, 47 (62\%) were aneuploid and 29 (38\%) diploid. All aneuploid cases showed a higher DNA cell content, with a mean DNA index of $1.63 \pm 0.46$ (range: 1.08-2.92). In most of the DNA aneuploid cases (81\%), a single aneuploid population was detected, while in 9 cases $(19 \%)$ more than one tumour stem cell clone was detected. The distribution of tumour cells along the different cell cycle phases was as follow: G0/G1, 85.4 $\pm 11.9 \%$; S, $10.9 \pm 9.8 \%$; and $\mathrm{G} 2 / \mathrm{M}, 3.5 \pm 3.2 \%$. The clinical and biological characteristics of the disease according to the existence of DNA aneuploidy are shown in Table 1. As may be seen, the aneuploid cases had a significantly higher ( $p=0.0001$ ) proportion of S-phase cells as compared to the diploid tumours. In addition, a higher proportion of deaths $(p=0.01)$ was observed among the cases displaying DNA aneuploidy. By contrast, no significant differences were observed between diploid and aneuploid cases with respect to age, sex, initial symptoms, degree of local infiltration, lymph node involvement, extranodal metastases, the TNM stage and the histological type.

Upon grouping the patients according to the percentage of S-phase cells into a low and a high proliferative groups (Table 2), no significant differences were observed for any of the clinical and pathological variables analysed except for a higher proportion of cases displaying DNA aneuploidy $(p=0.0001)$ among patients with high numbers of S-phase cells.

Table 1

Gastric cancer: Clinical, pathological and biological didease characteristics according to the presence of DNA aneuploidy by flow cytometry

\begin{tabular}{|c|c|c|c|}
\hline & $\begin{array}{c}\text { DNA diploid } \\
\text { cases }(n=29)\end{array}$ & $\begin{array}{l}\text { DNA aneuploid } \\
\text { cases }(n=47)\end{array}$ & $p$ value \\
\hline Age (years) ${ }^{*}$ & $70 \pm 10$ & $71 \pm 10$ & NS \\
\hline Sex $(\text { male/female ratio) })^{* *}$ & $62 / 38$ & $61 / 39$ & NS \\
\hline Tumour-related symptoms ${ }^{* *}$ & 56 & 44 & NS \\
\hline \multicolumn{4}{|l|}{ Depth of infiltration ${ }^{* *}$} \\
\hline $\mathrm{T} 1$ & 0 & 5 & \\
\hline $\mathrm{T} 2$ & 4 & 28 & \\
\hline $\mathrm{T} 3$ & 36 & 64 & 0.01 \\
\hline $\mathrm{T} 4$ & 60 & 0 & \\
\hline Lymph node involvement ${ }^{* *}$ & 68 & 95 & 0.001 \\
\hline Extranodal metastases ${ }^{* *}$ & 16 & 21 & NS \\
\hline \multicolumn{4}{|l|}{ Stage } \\
\hline I & 0 & 2 & \\
\hline II & 32 & 34 & NS \\
\hline III & 52 & 41 & \\
\hline IV & 16 & 21 & \\
\hline \multicolumn{4}{|l|}{ Histological type** } \\
\hline Well/moderately differentiated & 48 & 64 & NS \\
\hline Poorly differentiated & 52 & 36 & \\
\hline S-phase cells ${ }^{* *}$ & $6.0 \pm 4.4$ & $18.4 \pm 11.5$ & 0.0001 \\
\hline Individuals alive ${ }^{* *}$ & 68 & 36 & 0.01 \\
\hline
\end{tabular}

${ }^{*}$ Results expressed as means \pm standard deviation and ${ }^{* *}$ percentage of patients. NS, statistically non-significant $(p>0.005)$. 
Table 2

Gastric cancer: Clinical, pathological and biological didease characteristics according to the proliferative rate of tumour cells

\begin{tabular}{lccc}
\hline & $\begin{array}{r}\text { S-phase }<15 \% \\
\text { cases }(n=51)\end{array}$ & $\begin{array}{c}\text { S-phase }>15 \% \\
\text { cases }(n=25)\end{array}$ & $p$ value \\
\hline Age (years) & $70 \pm 10$ & $69 \pm 10$ & NS \\
Sex (male/female ratio) ${ }^{* *}$ & $66 / 34$ & $60 / 40$ & NS \\
Tumour-related symptoms** $^{* *}$ & 48 & 46 & NS \\
Degree of infiltration** & & & \\
T1 & 0 & 0 & \\
T2 & 7 & 0 & \\
T3 & 26 & 43 & NS \\
T4 & 67 & 56 & \\
Lymph node involvement** & 64 & 65 & NS \\
Extranodal metastases** & 17 & 26 & NS \\
Stage & & & \\
I & 2 & 4 & \\
II & 33 & 33 & NS \\
III & 47 & 37 & \\
IV & 16 & 25 & \\
Histological type** & & & \\
Well/moderately differentiated & 59 & 56 & NS \\
Poorly differentiated & 41 & 96 & \\
DNA ploidy** & 40 & 96 & 0.0001 \\
Individuals alive** & 52 & 42 & NS \\
\hline
\end{tabular}

${ }^{*}$ Results expressed as means \pm standard deviation and ${ }^{* *}$ percentage of patients. NS, statistically non-significant $(p>0.05)$.

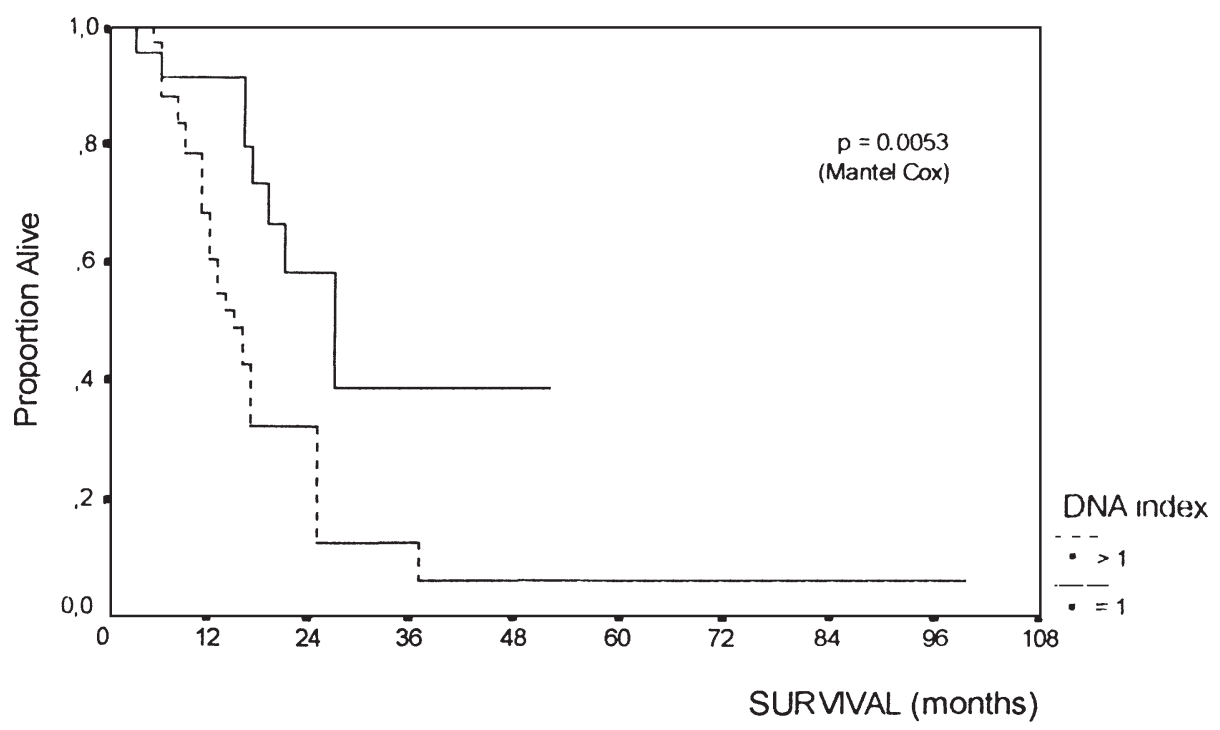

Fig. 1. Overall survival of patients with gastric cancer according to the presence of DNA aneuploidy (univariate analysis of prognosic factors). 
Analysis of the prognostic value of DNA measurements (Fig. 1) showed that cases displaying DNA aneuploidy are associated with a significantly lower survival $(p=0.005)$ as compared to the diploid patients. By contrast, in the present study the proportion of S-phase cells was of no value for predicting overall survival.

Multivariate analysis confirmed that the presence of DNA aneuploidy is an independent prognostic factor in gastric cancer $(p=0.003)$ together with the histological type $(p=0.03)$ and the existence of extranodal metastases $(p=0.05)$, these three parameters representing the best combination of disease characteristics for predicting survival in our series of gastric cancer patients.

\section{Discussion}

Several papers have been published in which DNA ploidy was analyzed in gastric cancer patients, the incidence of aneuploid cases ranging from 34 to 89\% [2,31]. Most of these studies were performed retrospectively on paraffin-embedded tissues $[4,9,14]$, and a few have been performed on fresh tumour speciments. On most occasions the relationship between the presence of DNA aneuploidy and other prognostic factors has been analyzed [11,16,18,28,34,36,37], but its value for predicting survival has not been extensively explored; actually, to the best of our knowledge, only Ohyama et al. [24,25] performed a multivariate analysis of prognostic factors in fresh tumour samples in which the independent value of DNA aneuploidy with respect to other well-established prognostic factors was explored. Interestingly, results described in these two publications by the same group are contradictory as regards the prognostic value of DNA aneuploidy.

In the present study, we followed the recommendations of the DNA Cytometry Consensus Conference held in October 1992 using an objective and reproducible technique for the measurement of cell DNA contents. The overall incidence of DNA aneuploidy was $62 \%$, which is similar to that found in other series $[15,16,23,28,33,38]$. Of these patients $19 \%$ displayed more than one DNA aneuploid population.

An association between the presence of DNA aneuploidy and the existence of lymph node involvement has previously been reported in gastric cancer patients [13,18,37]. This association is confirmed in the present series. By contrast, no clear association was found between the presence of DNA aneuploidy and other clinical and pathological findings such as age, sex, the existence of tumour-related symptoms at diagnosis, the disease stage or the degree of local infiltration. These findings are also in keeping with previous reports $[11,20]$. Similar to what has been reported by other groups $[6,20,28,36]$, no clear association was detected between the histologic type of the tumour and the presence of DNA aneuploidy, although a slightly higher incidence of aneuploid cases was found for well and moderately-differentiated carcinomas in agreement with the observations recently reported by several groups [7,12,28,29].

Although an association between the presence of DNA aneuploidy and the existence of extranodal metastases has been reported by other groups [16,37], these observations could not be confirmed in the present series.

Regarding the cell cycle distribution of tumour cells, a clear association was detected between the proportion of S-phase cells and the presence of DNA aneuploidy. Nevertheless, as pointed out previously by our group [26] and others [30], this could be related, at least partially, to technical questions associated with the lack of a specific indetification of tumour cells in the diploid cases. Accordingly, whereas in the aneuploid cases the assessment of the cell cycle distribution is specifically done for the tumour population, in diploid cases the final S-phase values strongly depend on both the proliferative activity of tumour cells and sample contamination by normal cells. 
Interestingly, no clear association was found between the proportion of S-phase cells and other clinical and pathological disease characteristics even when different S-phase cut-off values were used for diploid and aneuploid tumours.

The number of papers in which the cell cycle distribution of tumour cells has been explored is still scanty [9,24,37]. In some reports [25], a high S-phase fraction has been associated with a greater incidence of extranodal metastases and a more advanced stage of the disease. However, these findings have not been confirmed by others [11]. Although a slightly higher incidence of both extranodal metastases and stage IV was found in the present series for cases with a high proportion of S-phase cells, these differences did not reach statistical significance. Further technical developments in which specific indentification of tumour cells can be achieved are necessary to elucidate the real associations and the clinical value of S-phase measurements in gastric cancer.

Regarding overall survival, our results show that the presence of DNA aneuploidy is associated with both a higher incidence of deaths and a shorter median survival. In contrast, no prognostic value was found for the percentage of S-phase cells in spite of the association found between the ploidy status and the number of S-phase cells. These findings are in accordance with those reported by others $[4,13,18,24$, 37]. Interestingly, in a multivariate analysis of prognostic factors we observed that the presence of DNA aneuploidy is an independent prognostic factor and, together with the histologic type and the existence of extranodal metastases, represents the best combination of factors for predicting survival. To the best of our knowledge, this is the first report in which the independent prognostic value of DNA aneuploidy is clearly demonstrated in fresh tumour samples from gastric cancer patients. It could be argued that due to the high mean age of the patients included in the present study, overall survival rates could be similar to those expected for a normal age- and sex-matched population. However, it has previously been clearly show that age- and sex-matched normal individuals display a significantly higher likelihood of survival [21].

In summary, in the present study we show that in gastric cancer patients the presence of DNA aneuploidy by flow cytometry is an important prognostic factor, while the estimation of the clinical value of the S-phase fraction based on the measurement of cell DNA content is of no clear prognostic impact. Accordingly, the use of more accurate technical procedures for the specific identification of S-phase tumour cells is mandatory.

\section{Acknowledgements}

We would like to thank Pilar Paradinas and Esther Sanchez for their technical assistance.

\section{References}

[1] American Joint Committee on Cancer, Manual for Staging of Cancer, 3rd edn, J.B. Lippincott, Philadelphia, 1988, pp. 6974.

[2] G. Baretton, O. Carstensen, M. Schardey and U. Lohrs, DNA ploidy and survival in gastric carcinomas: a flow-cytometric study, Virchows Arch. Path. Anat. 418 (1991), 301-309.

[3] M.J. Brito, M.I. Filipe, G.T. Williams, H. Thompson, M.G. Ormerod and J. Titley, DNA ploidy in early gastric carcinoma (T1): a flow cytometric study of 100 European cases, Gut 34 (1993), 230-234.

[4] I. D'Agnano et al., DNA ploidy, proliferative index and epidermal growth factor receptor: Expression and prognosis in patients with gastric cancer, Lab. Invest. 72 (1995), 432-438.

[5] Y. Esaki, R. Hirayama and K. Hirokama, A comparison of patterns of metastases in gastric cancer by histologic type and age, Cancer 65 (1990), 2086-2090. 
[6] M.I. Filipe, J. Rosa, A. Sandey, P.R. Imrie, M.G. Ormerod and W. Morris, Is DNA ploidy and proliferative activity of prognostic value in advanced gastric carcinoma?, Hum. Pathol. 22 (1991), 373-378.

[7] H.L. Flyger, I.J. Christensen, J. Thorup, T.V. Hakansson and T. Norgaard, DNA aneuploidy in gastric carrcinoma. Flow cytometric data related to survival, location and histopathologic findings, Scand. J. Gastroenterol. 30 (1995), 258-264.

[8] R. Garsia Sanz et al., Prognostic implications of DNA aneuploidy in 156 untreated multiple myeloma patients, $B r . J$. Haemat. 90 (1995), 106-112.

[9] D.W. Hedley, M.L. Friedlander, I.W. Taylor, C.A. Rugg and E.A. Musgrove, Method for analysis of cellular DNA content of paraffin-embedded pathological material using flow-cytometry, J. Histochem. Cytochem. 31 (1983), 1333-1335.

[10] T. Hirota, A. Oakioi, M. Itabashi and K. Maroyama, Significance of histological type of gastric carcinoma as a prognostic factor, Stomach and Intestines 26 (1991), 1149-1158.

[11] K. Hyung Lee et al., DNA flow cytometry of stomach cancer. Prospective correlation with clinicopathologic findings, Cancer 72 (1993), 1819-1826.

[12] M. Ikeguchi et al., A relationship between the cell proliferative activity of gastric cancers and that of the normal epithelium, Anticancer Res. 15 (1995), 821-825.

[13] H.J. Johnson, C. Bellucco, S. Massood, A.M. Abou-Azama, L. Kahn and L. Wise, The value of flow cytometric analysis in patients with gastric cancer, Arch. Surg. 128 (1993), 314-317.

[14] O.P. Kallioniemi, Comparison of fresh and paraffin-embedded tissue as starting material for DNA flow cytometry and evaluation of intratumor heterogeneity, Cytometry 9 (1988), 164-169.

[15] H. Kimura et al., Implication of flow cytometry in preoperative detection of biologic variables of gastric cancer and malignant condition of gastric remnant cells obtained by endoscopic biopsy, Oncology 51 (1994), 479-484.

[16] H. Kimura and Y. Yonemura, Flow cytometric analysis of nuclear DNA content in advanced gastric cancer and its relationship with prognosis, Cancer 67 (1991), 2588-2593.

[17] H. Kimura et al., Proliferative activity in gastric cancer with Ki67 and propidium iodide: analysis by flow cytometry, Nippon Geka Gakkai Zasshi 93 (1992), 388-392.

[18] D. Korenaga, T. Okamura, A. Saito, H. Baba and K. Sugimachi, DNA ploidy is closely liniked to tumor invasion, lymphnode metastasis and prognosis in clinical gastric cancer, Cancer 62 (1988), 309-319.

[19] J.Y. Li, L.R. Lu and S.X. Zhang, Relation of DNA content to biological characteristics and prognosis in advanced gastric carcinoma, Chung Hua Ping Li Hsuch Tsa Chih 23 (1994), 135-137.

[20] J.C. Macartney, R.S. Camplejohn and G. Powell, DNA flow cytometry of histological material from human gastric cancer, J. Pathol. 148 (1986), 273-277.

[21] Ministerio de Sanidad y Consumo, Indicadores de salud, Programa Regional Europeo, Salud para todos, 1995.

[22] K. Nakamura, T. Veyama, T. Yao, Z.X. Xuan, K. Ambe and Y. Adachi, Pathology and prognosis of gastric carcinoma, Cancer 70 (1992), 1030-1037.

[23] K. Nishida et al., Flow cytometry analysis of nuclear DNA content in the endoscopic biopsy tissues of gastric cancer, Am. J. Clin. Oncol. 18 (1995), 325-330.

[24] S. Ohyama, Y. Yonemura and I. Miyazaki, Prognostic value of S-phase fraction and DNA ploidy studied with in vivo administration of bromodeoxyuridine on human gastric cancer, Cancer 65 (1990), 116-121.

[25] S. Ohyama, Y. Yonemura and I. Miyazaki, Proliferative activity and malignancy in human gastric cancers, Cancer 69 (1992), 314-321.

[26] A. Orfao et al., A new method for the analysis of plasma cell DNA content in multiple myeloma samples using a CD38/ propidium iodide double staining technique, Cytometry 17 (1994), 332-339.

[27] J.I. Paz Bouza et al., Transrectal fine needle aspiration biopsy of the prostate combining cytomorphologic, DNA ploidy status and cell cycle distribution studies, Pathol. Res. Pract. 190 (1994), 682-689.

[28] M. Rugge et al., Pathology and ploidy in the prognosis of gastric cancer with No extranodal metastasis, Cancer 73 (1994), $1127-1133$.

[29] O. Sasaki, K. Soejima and T. Haraguchi, Intratumor DNA ploidy distribution pattern and its relation to histologic type in gastric carcinoma, Pathol. Res. Pract. 188 (1992), 545-549.

[30] T.V. Shankey et al., Guidelines for implementation of clinical DNA cytometry, Cytometry 14 (1993), $472-477$.

[31] L. Teodori et al., Cytometrically-determined relative DNA content as an indicator of neoplasia in gastric lesions, Cytometry 5 (1984), 63-70.

[32] R.M. Thomas and L.H. Sobin, Gastrointestinal cancer, Cancer 75 (1995), 154-170.

[33] S. Tomisaki et al., DNA ploidy pattern and p53 over expression in gastric cancer, Gan To Kagaku Kyoho 22 (1995), $115-117$.

[34] M. Victorzon, P.J. Robert, C. Haglund, K. von Boguslawsky and S. Nordling, Ki-67 immunoreactivity, ploidy and S-phase fraction as prognostic factor in patients with gastric carcinoma, Oncology $\mathbf{5 3}$ (1996), 182-191.

[35] L.L. Vindelow, I.J. Christensen and N.I. Nissen, A detergent-trypsin method for the preparation of nuclei for flow cytometric DNA analysis, Cytometry 3 (1983), 323-327. 
[36] J.I. Wyatt, P. Quirke, D.C. Ward, A.D. Clayden, M.J. Dixon and D. Johnston, Comparison of histopathological and flow cytometric parameters in prediction of prognosis in gastric cancer, J. Pathol. 158 (1989), 195-201.

[37] Y. Yonemura, S. Ohyama, K. Sugiyama, T. Kamata, X. de Aretxabala and H. Kimura, Retrospective analysis of the prognostic significance of DNA ploidy patterns and S-phase fraction in gastric carcinoma, Cancer Res. 50 (1990), 509514.

[38] L.F. Zuo, Analysis of DNA ploidy in 3,050 cases of malignant tumors, Chung Hua Ping Li Hsueh Tsa Chih 21 (1992), $167-170$. 


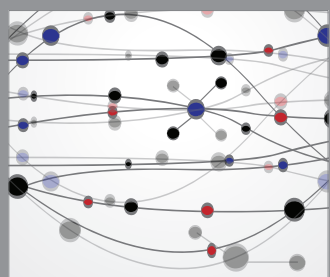

The Scientific World Journal
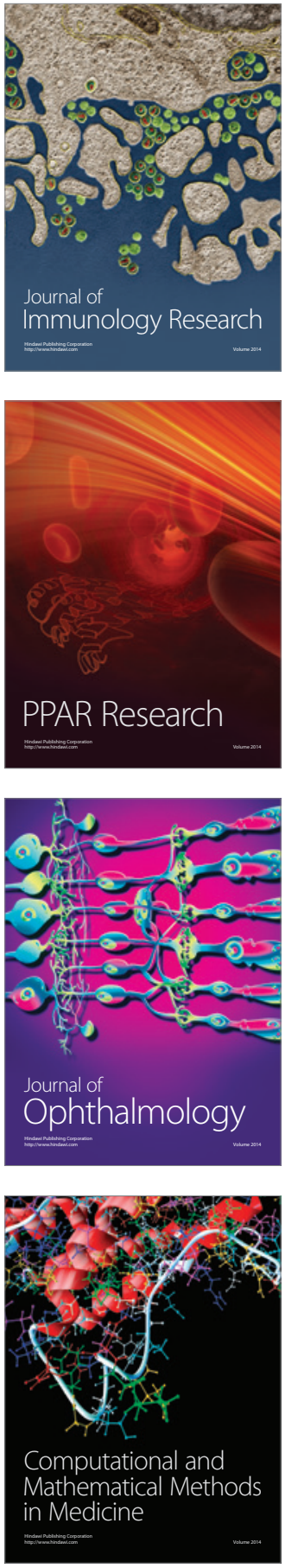

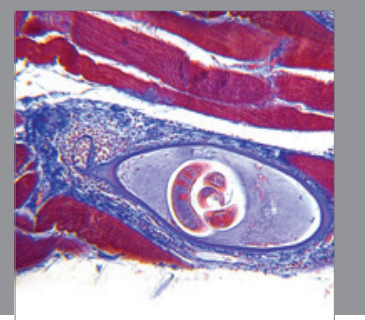

Gastroenterology

Research and Practice
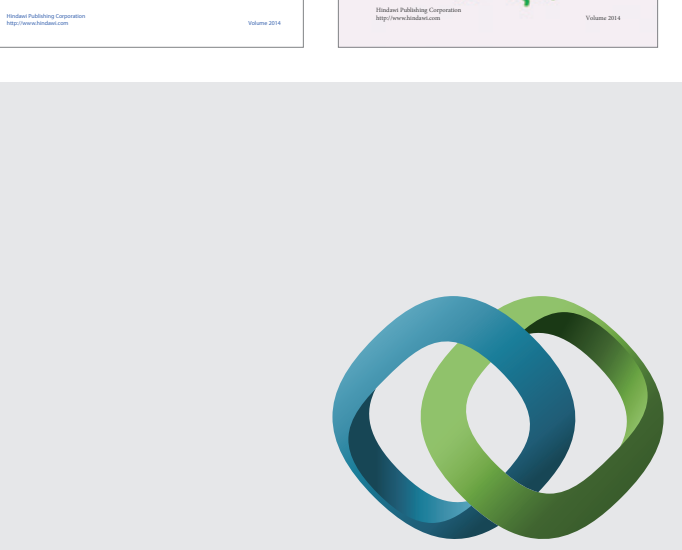

\section{Hindawi}

Submit your manuscripts at

http://www.hindawi.com


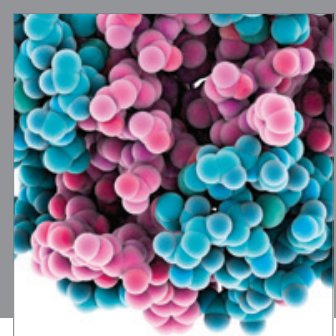

Journal of
Diabetes Research

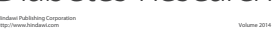

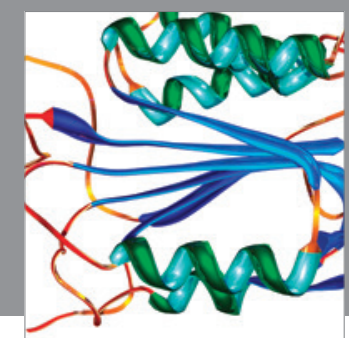

Disease Markers
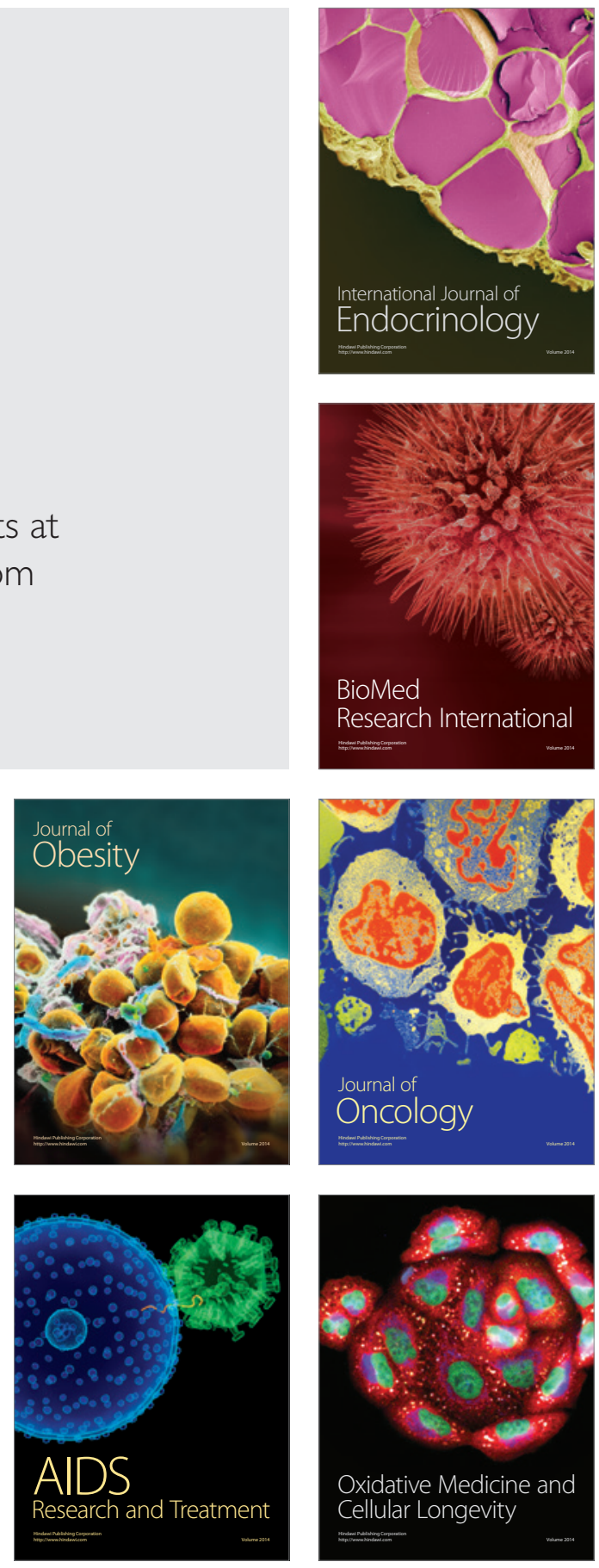